\title{
Approaches to the Management of Presumed Immediate Hymenoptera Venom Allergy and Non-Detectable IgE
}

\author{
Ervin Ç. Mingomataj ${ }^{1,2, *}$ and Alketa H. Bakiri \\ 1 “Mother Theresa” School of Medicine, Dept. of Allergology \& Clinical Immunology, Tirana - Albania \\ ${ }^{2}$ University of Tirana, Nursing Faculty, Dept. of Preclinical Disciplines, Tirana - Albania
}

\begin{abstract}
:
Objective: To provide a comprehensive evaluation in patients with a convincing history of immediate insect allergy but negative skin test and/or specific IgE results, adequately addressing the question of how best to manage them.

Data sources: Observational peer-reviewed studies and case reports were searched on Pub-Med database from 1998 up to March 2009 using the following keywords: Hymenoptera Allergy \& Negative IgE (Negative Skin Tests).

Study selection: Studies on supplemental diagnostic tests that provided data from patients with immediate hymenoptera allergy but negative conventional tests results to the offending allergens were selected. In this work, we also included studies providing additional relevant information regarding this issue.

Results: Among 43 identified papers only 9 of them presented relevant original data, while the other papers were reviews. In the majority of the cases, the culprit insect was identified with in vitro tests such as Basophil Activation Test, Cellular Allergen Stimulation Test or Western blot, whereas in vivo (less frequently) with sting challenge or dialyzed venom skin test.

Conclusions: The management of patients with a convincing history of immediate insect allergy but negative conventional test results requires an adaption of the guidelines including an incorporation of the novel diagnostic tools. Although cellular tests represent equivalent sensitivity and superior specificity as compared with standard ones, these tests still remain supplementary diagnostic tools. In a minority of cases (especially in the developing countries where cellular tests cannot be performed), venom immunotherapy in adult subjects could be taken into account based solemnly on the history of a clear patient's identification of the culprit insect.
\end{abstract}

Keywords: Hymenoptera venom allergy, negative skin tests, negative specific IgE, sting challenge test, cellular activation tests.

\section{INTRODUCTION}

Immunoglobulin (Ig) E-mediated hymenoptera venom (HV) allergy is an important health problem and the correct diagnosis is a prerequisite for an effective management with specific immunotherapy (SIT) [1]. Consequently, the diagnosis should be based on more than one confirmatory test [1]. The indication for SIT is based on history but also on current respective diagnostic procedures such as intradermal tests, specific IgE determination and an optional sting challenge test $[2,3]$.

Despite performing the aforementioned tests, the culprit insect may remain unidentified [3-6]. In other words, a minor proportion of allergic patients to hymenoptera stings show negative results to usual tests (especially during skin prik testing). Actually, current practice guidelines do not adequately address the question of how to best manage the patient presenting with a convincing history of $\mathrm{HV}$ allergy

*Address correspondence to this author at the Rruga Myslym Shyri, P. 47, Apt. 15, Tirana - Albania; Tel: 00355682621913; Fax: 0035542229203; E-mail: allergology@gmx.de who show negative skin tests (ST) and/or specific IgE results $[6,7]$. While the aforementioned documents suggest that SIT should be performed only in patients with proven IgEmediated systemic reactions, opinions on the diagnosis and treatment of patients with systemic reactions who lack a specific IgE response are quite different $[3-5,7,8]$. In this context, most allergists consider ST to be highly accurate and interpret a negative result as an absence of a clinically relevant $\mathrm{HV}$ sensitization [9]. In contrast to them, $8 \%$ of British SIT-practicing clinicians commence SIT in patients with negative specific IgE and a severe reaction history [10].

The lack of a guideline that could supplement the imperfect diagnostic armamentarium and the conduction of the SIT after severe reactions in patients with negative specific IgE can lead to the opinion that diagnostic procedures need an improvement [11]. Generally, in the diagnosis of an immediate allergy are used allergen extracts that allow the identification of the allergen-containing source responsible for respective allergic symptoms but not the disease-eliciting molecules [12]. With the recent introduction of recombinant pneumoallergens, a large panel of allergenic molecules has become available [12]. In the future, this strategy may result 
also helpful in the diagnosis of HV allergy [13]. Diagnostic methods would be improved through the use of such allergens which, being "uncontaminated" by other venom constituents, allow for a precise setup of an "individual allergogram" [11]. Preliminary trials analyzing diagnostic tests using dialyzed or recombinant allergens seem to be promising: the specificity is clearly increased in both skin testing and in determining specific $\operatorname{IgE}$ antibodies when compared to the natural extracts $[14,15]$. Furthermore, the purified HV extracts are introduced in various cellular activation tests as novel diagnostic tools [1-3]. The recently published studies focusing on the diagnostic effectiveness of these tests have forced researchers to reexamine this important management issue and consider the role of in vitro testing in the management of the HV allergy [9]. Based on recent publications, we aimed to review the current status of knowledge concerning the management of subjects with a convincing history of immediate HV allergy but negative venom ST and/or specific IgE results, suggesting consideration of this issue in future working guidelines.

\section{OBJECTIVE AND STUDY SELECTION}

Objective: To provide comprehensive guidelines for the treatment of patients with a convincing history of insect venom allergy but negative venom ST and/or specific IgE results.

Data sources: Observational peer-reviewed studies, review articles, and case reports were searched on Pub-Med database from 1998 up to March 2009 using the following keywords: Hymenoptera Allergy \& Negative IgE (or Negative Skin Tests).

Study selection: Original studies providing data from patients with an immediate HV allergy, but negative venom ST and/or negative specific IgE to the offending allergens were selected. Relevant reviews for the issue were included in the introduction or discussion.

The scanned literature: During the last decade many publications are focused on the practical management of the HV allergic patients. However, our focused searching on PubMed identified only 43 papers published in the mentioned period of time. Only 9 of them (one casuistic) resulted as appropriate original interventional studies [1-3, 8, 15-19]. The including criteria was the use of additional diagnostic tools for the identification of culprit insect in patients with a convincing history of immediate HV allergy but negative ST and/or specific IgE to the offending allergens (see the Table 1). In 6 of them the supplemental diagnostic tool consisted of one or more in vitro tests, in 2 trials a sting challenge was performed, and in the last case ST were conducted with dialyzed yellow jacket venom [1-3, 8, 15-19]. In general, in these trials we identified 122 subjects with negative response for at least one of the usual tests, and 75 further subjects with negative result for both tests (for more information, see the Table 1). The rest of the identified original papers were excluded, because they did not meet the inclusion criteria, while relevant reviews were appropriately used in the introduction or discussion $[4-7,9,10,13,20-46]$. Thus, some papers were focused on the venom double-positivity or on the question of false positive results due to irrelevant venom carbohydrates $[13,29,33,35,41]$. While the topic in a few studies was the asymptomatic sensitization, some additional studies addressed the question of ST or specific IgE negativation after SIT [26, 32, 34, 36, 37, 39, 40, 42, 43]. Further papers analyzed the cross-reactivity between hymenoptera and non-hymenoptera allergens or the ST-reproducibility [9, $22,23,30,38]$. Two additional original studies reported about subjects with negative usual test responses, but both were not included for the following reasons: the first paper had as topic the anaphylaxis etiology among children (but no one had shown negative standard results after episode of HV anaphylaxis), while the second one reported about a negative specific IgE response to the HV after a fatal insect sting [4, 46]. The last exclusion was a report about a European subject allergic to fire ants, but with negative response to the rest of the HV [28].

\section{RESULTS}

Purified venom in skin tests: The dialysis of $\mathrm{HV}$ extracts removes low molecular-weight amines and irritants that contribute to the false-positive ST reactions at higher concentrations [15]. In fact, the dialyzed venoms are routinely used in vitro assays and for skin testing in Europe. A trial included in this review compared the diagnostic ranges and clinical detection rates of ST using dialyzed and crude yellow jacket venom, particularly in patients with negative ST results. The intradermal ST with dialyzed venom resulted positive up to $10 \mu \mathrm{g} / \mathrm{ml}$ or less in $79 \%$ of 24 patients with a positive history but negative ST reactions using undialyzed venom [15]. In particular, four out of seven patients who showed negative $\mathrm{IgE}$ response to yellow jacked venom turned positive after dialyzed venom testing (see the Table 1) [15]. These results were unlikely to be false-positive, because three out of four patients with negative response to undialyzed venom experienced also systemic reaction after sting challenge. These findings indicate that the use of dialyzed venom may improve the ST accuracy for the detection of yellow jacket allergy and may be subject of further studies [15].

Cellular/in vitro tests: In the recent years, 2 new and promising cellular tests have been introduced: the sulfidoleukotriene (LT) release by basophils, assessed by ELISA (CAST-ELISA) and the activation of the basophilic CD63 marker as measured by flow cytometry (Flow-CAST). The latter test is also known as basophil activation test (BAT) [3, 5]. CAST-ELISA assesses the in vitro LT release from blood leukocytes of presumably allergic patients upon stimulation with various allergen doses [3]. Flow-CAST investigates the allergen-induced in vitro basophils' activation as determined by the demonstration of the CD63 molecule on the basophil membrane $[1,3,5]$. CD63 is normally expressed on the inside of vesicle membranes. Under membrane-bound IgE cross-linking, the fusion of the vesicle to the plasma membrane allows the CD63 expression on the external membrane and can then be detected by flow cytometry $[1,3]$. Several reports have proved their potential role in various immediate allergies.

In particular, the usefulness of the cellular assays CASTELISA and Flow-CAST in the management of the HV allergy was investigated respectively in 3 and 5 patient control studies selected in this review [1-3, 16-18]. In the CASTELISA studies were included 35 subjects with negative responses for at least one of the standard tests, and 2 additional 
Table 1. The Comparative Analysis of Supplemental Tests in The Diagnosis of Hymenoptera Allergic Patients with Negative Usual Test Responses

\begin{tabular}{|c|c|c|c|c|c|c|c|}
\hline $\begin{array}{l}\text { Supplemental Test } \\
\text { (SDT) [ref] }\end{array}$ & Venom Type & $\begin{array}{l}\text { Patients } \\
\text { (n) }\end{array}$ & $\begin{array}{l}\text { Subjects with Nega- } \\
\text { tive Usual Test } \\
\text { Responses [Skin } \\
\text { Tests / IgE / Both] }\end{array}$ & $\begin{array}{c}\text { General } \\
\text { SDT- } \\
\text { Sensitivity } \\
(\%)\end{array}$ & $\begin{array}{c}\text { SDT-Sensitivity (\%) } \\
\text { [When Usual Tests } \\
\text { Negative, Skin Tests } \\
\text { / IgE / Both] }\end{array}$ & $\begin{array}{l}\text { General } \\
\text { SDT- } \\
\text { Specificity } \\
(\%)\end{array}$ & Disadvantages \\
\hline Western blot [18] & $\begin{array}{l}\text { Yellow } \\
\text { Jacked }\end{array}$ & 30 & $4 / 3 / 0$ & $100 \%$ & $100 \% / 100 \% /-$ & $70 \%$ & $\begin{array}{l}\text { Costly, not } \\
\text { prognostic for } \\
\text { future stings }\end{array}$ \\
\hline Flow-CAST $[1-3,16-17]$ & $\begin{array}{l}\text { Bee \& } \\
\text { Yellow } \\
\text { Jacked }\end{array}$ & 368 & $27 / 25 / 11$ & $\begin{array}{l}81.4- \\
100 \%\end{array}$ & $\begin{array}{c}0-100 \% / 86-100 \% / \\
100 \%\end{array}$ & $86-100 \%$ & $\begin{array}{l}\text { Costly, not } \\
\text { prognostic for } \\
\text { future stings }\end{array}$ \\
\hline CAST-ELISA [2-3, 17] & $\begin{array}{l}\text { Bee \& } \\
\text { Yellow } \\
\text { Jacked }\end{array}$ & 189 & $15 / 20 / 2$ & $85.7-100 \%$ & $\begin{array}{c}86-100 \% / 100 \% / 50- \\
100 \%\end{array}$ & $95-100 \%$ & $\begin{array}{l}\text { Costly, not } \\
\text { prognostic for } \\
\text { future stings }\end{array}$ \\
\hline Challenge test $[8,19]$ & Bee \& Wasps & 308 & $43 / 0 / 57$ & $21.7 \%$ & $24.3 \% /-/ 14.3 \%$ & & $\begin{array}{c}\text { Safety, needed } \\
\text { equipments }\end{array}$ \\
\hline Dialyzed venom [15] & $\begin{array}{l}\text { Yellow } \\
\text { Jacked }\end{array}$ & 44 & $17 / 0 / 7$ & $88.6 \%$ & $79.2 \% /-/ 57.1 \%$ & $100 \%$ & $\begin{array}{l}\text { Lower sensitiv- } \\
\text { ity }\end{array}$ \\
\hline
\end{tabular}

Legend: SDT - supplemental diagnostical test used; (-) - non-evaluated in the original work. The fourth column separately shows patients (n) with only with negative skin test response, only negative IgE, and with negative response for both mentioned tests respectively. In the same separation manner, the SDTsensitivity $(\%)$ is shown on the sixth column for all 3 mentioned subgroups.

subjects with negative results for both usual methods (see the Table 1). In the Flow-CAST (BAT) studies we identified 52 allergic subjects with negative response for at least one of the usual tests, and 11 additional allergics with negative result for both tests (see the Table 1). The results of these tests were compared with ST, specific IgE, or basophil histamine release test (BHR) results, while the positive history served as reference. The general sensitivity of the cellular assays (81-100\%) was comparable to usual tests, and in three trials the combination of ST, specific IgE and cellular assay(s) yielded a positive predictive value of $100 \%$ for both venoms [1, 16, 17]. Notably, in a study BAT represented also a higher general sensitivity as compared to usual tests [17]. In addition, all trials demonstrated that relative specificities were considerably higher for both cellular assays (FlowCAST 86-100\%, CAST-ELISA 95-100\%) allowing also the detection of the culprit insect in patients with reactivity to both insects [1-3, 16, 17]. In a survey, flow cytometric analysis demonstrated a significant increase of the number of CD63 positive cells while all controls were negative [17]. In summary, these findings indicated that both tests could be valuable tools for the identification of culprit insect in patients with unclear clinical history $[1,3]$.

According to a survey conducted in 14 patients, the mentioned methods were more effective than basophil histamine release test (BHR) in difficult cases of the HV allergy, where history, ST and specific IgE determination do not allow a clear decision regarding the SIT [2]. The concordance between BAT and CAST-ELISA (78.6\%) was more evident as compared between BAT and other tests, such as specific IgE, BHR or ST (42.9-69.1\%). However, despite their perceived usefulness, they did not give additional diagnostic advice in a few cases.
A further in vitro technique recently used in the diagnosis of HV allergy is the Western blot [18]. In this method allergen extracts consist of a complex mixture of proteins which can be separated by molecular size on SDS-PAGE. Three major allergens can be identified in the yellow jacked venom, namely phospholipase $\mathrm{A} 1$, hyaluronidase and antigen 5 [18]. Following electrophoretic transfer of the separated proteins onto nitrocellulose membranes, incubation of these matrices with patient sera reveals the specific antibody pattern of an individual. Based on these findings, Zollner et al. evaluated the Western blot sensitivity and specificity as a possible alternative to specific IgE serum analyses in 30 patients with generalized reaction following the yellow jacked sting [18]. Seven out of these subjects showed negative response for the ST or specific IgE (see the Table 1). The Western blot sensitivity reached $100 \%$ in the samples studied showing superiority to the specific $\operatorname{IgE}(90 \%)$ or ST $(87 \%)$. When analyzing the appearance of a specific band for antigen 5 or hyaluronidase, the Western blot inhibition specificity and overall diagnostic value was the most efficient test (specificity $97 \%$ and $100 \%$, overall efficiency $96.8 \%$ and $93.2 \%$, respectively). These data indicate that this technique may be also a helpful diagnostic tool during allergy to the yellow jacked venom [18].

Summarizing the data of all the six in vitro trials, the mentioned tests demonstrate an equivalent sensitivity to the standard ones $[1-3,16-18]$. In contrast, the relative specificities and maybe, the overall efficiencies seem to be superior to them $[1-3,5,16-18]$. Despite the lower total specificity reported by Zollner et al., (see the Table 1) the specificity and Western blot overall efficacy for specific bands of hyaluronidase and antigen 5 in the wasp allergy diagnosis was equally excellent to other in vitro tests $[3,5$, 18]. The reported data indicate that cellular tests may be an 
additional diagnostic tool of choice, when standard tests do not identify the culprit insect $[1,3,16]$. The general BAT sensitivity reached a positivity rate of $90 \%$ in a limited number of patients with double-negativity result for standard tests $[1-3,16,18]$. Similar sensitivity rates for in vitro tests are reported for patients with mono-positivity for standard tests, but evident differences between authors have been also reported [1-3, 16-18]. In particular, in three out of five studies concerning the BAT, authors reported the identification of culprit insect in all cases with negative ST and specific IgE responses [1, 16, 17]. For example, Ebo et al. reported that BAT had an excellent sensitivity in all 7 patients with double-negativity, but a sensitivity rate of only $80-86 \%$ in patients with mono or double positive response to usual tests [1]. Taken together, these findings indicate that a combination of standard and cellular tests may reach maximal sensitivity rates, and in vitro tests could be useful, if additional information to standard testing is needed $[1,3,16]$. Despite reported variations, the overall diagnostic efficacy of such tests like BAT, CAST-ELISA in HV allergy and Western blot (at least during the yellow jacked venom allergy) seem to be comparable to each other [1-3, 16-18]. With respect to differences on the diagnostic effectiveness between hymenoptera species, the data were less conclusive, as long as in the majority of studies the study contingent allergic to the honeybee venom was too limited. However, in two studies with a representative proportion of honeybee allergic subjects there were no differences in the diagnostic value between honeybee and yellow jacked venom patients $[3,16]$.

The sting challenge test: An optional diagnostic tool of $\mathrm{HV}$ allergy is the sting challenge test [8, 19]. This test is used only in limited cases of sting reactions for the confirmation of HV allergy when conventional tests revealed no conclusion [19]. Zidarn et al. presented a patient with a history of atypical systemic reaction after a bee sting (see the Table 1) [8]. ST and IgE for the bee venom were negative. The sting challenge test resulted positive, while mast cell activation was proved by tryptase level elevation [8]. Afterwards, the patient was treated with SIT.

Based on the reports about patients with negative venom ST responses but severe reactions to subsequent stings, Golden et al. recently underwent a prospective study aiming to increase awareness about the patients with negative ST to $\mathrm{HV}$, and to determine the frequency and significance of such ST responses in subjects with a history of systemic reactions [19]. Moreover, in patients who gave informed consent, a sting challenge was performed. Ninety-nine (32\%) out of 307 patients with positive histories screened with the sting challenge had negative intradermal venom ST responses, while $56(57 \%)$ of 99 patients with positive histories and negative $\mathrm{ST}$ responses had also negative specific IgE results (see the Table 1) [19]. The sting challenge was performed in 141 patients with positive ST responses and $30(21 \%)$ of them experienced systemic reactions. This test was also performed in 37 patients with negative ST responses and positive specific IgE, as well as in 14 patients with negative ST responses and specific IgE results (see the Table 1) [19]. Forty-nine of the 51 patients with negative ST responses underwent a sting challenge with yellow jacket, while the rest of them (2 patients) underwent a challenge with Polistes venom. Eleven subjects (22\%) with negative ST responses experienced a systemic reaction to the challenge sting: two of them (14\%) had negative IgE results, while the rest (9 subjects, 24\%) had positive IgE results only at $1 \mathrm{ng} / \mathrm{ml}$ (see the Table 1) [19]. These data indicate that, independently to the outcome of the IgE, ST responses can be negative in patients who may subsequently experience further systemic sting reactions [19]. Systemic reactions in patients with negative venom $\mathrm{ST}$ responses may reflect a limited ST diagnostic sensitivity for IgE-mediated hypersensitivity. Hence, the anaphylactic history after hymenoptera stings should not be ignored by the allergologist because of negative responses to standard tests [19]. The finding that 9 out of 11 subjects with negative ST response and systemic anaphylaxis after sting challenge have shown barely detectable IgE antibody levels may lead to the conclusion that such cases may be frequently associated with false-negative results when diagnostic procedures are performed in laboratories that lack qualitative materials and equipments [9, 19].

\section{DISCUSSION}

Based on the present understanding of HV allergy, the practicing allergologist is not infrequently faced with the dilemma of the ST or specific IgE negative patient [20]. At least $10 \%$ of patients with suspected HV allergy show negative results during usual testing [17]. A negative response to these tests indicates that such subjects are not at all allergic to HV. However, the hyperreactivity to the HV may remain unidentified after standard testing in a minority of them. These review aimed to elucidate the actual knowledge about the management of these cases. This knowledge may lead to an adaption of future guidelines.

First, the undetected allergic reactions should be distinguished from the reactions mediated by non-immunologic mechanisms [6]. Negative ST after hymenoptera sting reactions may include differential diagnoses such as panickingdisorders, grand-mal epilepsy, etc. Moreover, mastocytosis or "anaphylactoid" reactions has been reported with increasing frequency among the patients with negative ST [20]. To detect systemic mastocytosis, serum tryptase level must be analyzed in patients with a severe sting reaction history [6]. Even in subjects without a clinical evidence for mastocytosis, elevated serum tryptase levels are found in up to $30 \%$ of the patients with a severe shock history following hymenoptera sting [14, 44, 47]. Additional major causes of false negative results are changes in extract potency and the skin reactivity suppression after antihistamines intake [21, 48]. With regards to the negative specific IgE, many authors recommend to repeat the tests after several weeks because circulating $\mathrm{IgE}$ antibodies might be consumed by the allergic reaction [6, 22, 23, 48]. Rieger-Ziegler et al. reported that 12 of 13 patients with honeybee- and 14 of 18 patients with vespula venom sensitization had specific IgE classes 1 or higher within the first 2 weeks after the hymenoptera sting [22]. All five patients with negative specific $\operatorname{IgE}$ results within the first two weeks had detectable specific IgE concentrations a few weeks later. These findings indicate that specific $\operatorname{IgE}$ determination is in most cases useful even during the first two weeks after the sting [22]. This allows early decisions on further diagnostic procedures and the therapeutic way to 


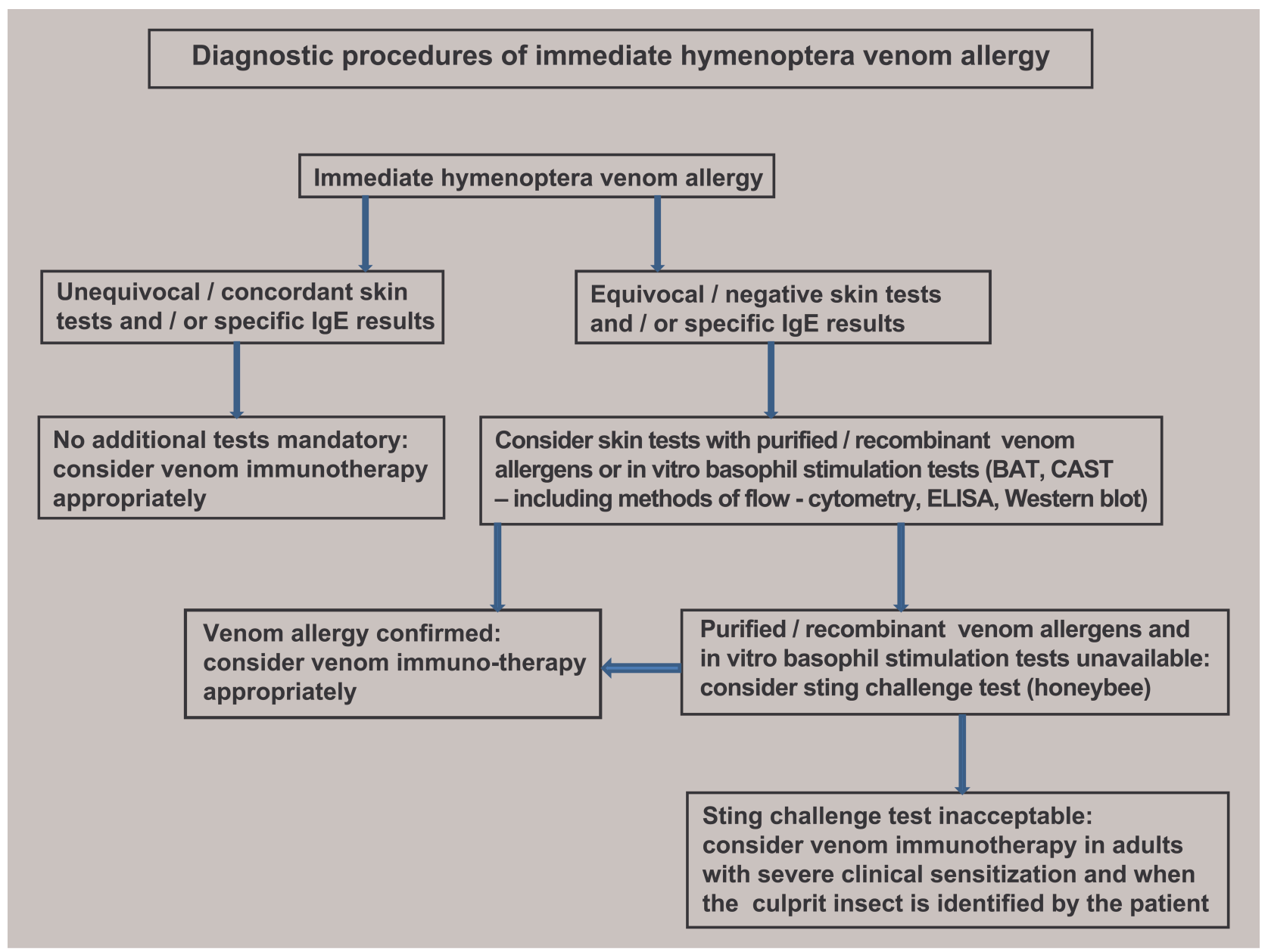

Fig. (1). As the hymenoptera sting patients have unequivocal and concordant specific IgE and venom skin test results, no additional diagnostic tests are mandatory. In the remaining patients, diagnosis can be complicated by entirely negative venom skin tests and specific IgE results. In the majority of such difficult cases (when systemic mastocytosis excluded), basophil activation test, cellular antigen stimulation test, western blot, and optionally, sting challenge test constitute useful complementary diagnostic instruments and contribute to the initiation of potentially life-saving specific immunotherapy. Meanwhile, if these tests cannot be performed, the introduction of specific immunotherapy could be taken into account, at least among adult patients with severe systemic clinical sensitization (even if conventional tests negative - for more information see the conclusion).

choose. Patients with no detectable specific IgE should, however, be retested within a few weeks $[9,19,21,22]$.

Recently, further strategies are established for the minimizing of undetected or difficult cases of HV allergy. Such strategies as the use of dialyzed venoms or recombinant allergens may lead to an improved diagnostic accuracy when compared to standard tests [13-15, 49]. Despite the lower specificity rate as compared to novel in vitro tests, the use of dialyzed allergens as standard diagnostic procedure can be regarded as the most convenient method with respect to costefficiency relationship $[1,5,15,16]$. Meanwhile, the use of specific IgE to both recombinant allergens Api $\mathrm{m} 1$ and Ves v5 distinguish a true double sensitization from the irrelevant cross-reacting of HV carbohydrate determinants [13].

In spite of the mentioned methods, cellular tests could be included into the practice parameter guidelines to support a positive clinical history during the diagnostic process $[1,6$,
7, 24]. Recent evidence has demonstrated that Flow-CAST technique was decisive in patients with completely negative specific IgE and ST results [1, 16]. A further equally helpful in vitro test in the identification of the culprit insect is the Western blot $[5,18]$. Its superior specificity and overall efficiency in comparison to the usual tests makes this method a valuable diagnostic tool [18]. Nevertheless, these tests are only supplementary tools that should be used in difficult diagnostic cases (see the Fig. 1) $[11,48,50]$. This is because neither in vivo tests, nor in vitro ones have optimal sensitivity; so, both sensitive methods might be required in such cases $[1,16]$. In addition, in vitro tests are expensive as compared to ST with purified HV [15].

In about $15 \%$ of subjects allergic to $\mathrm{HV}$, basophils do not release histamine in the presence of antigen [25, 51]. Consequently, the clinical history should drive the diagnosis of insect sting allergy and the indication for SIT [48]. A concrete diagnostic tool with a certain similarity to clinical his- 
tory can be the sting challenge test $[8,19,52]$. In fact, only a minority of patients with positive history for systemic anaphylaxis and negative standard tests' results also experienced a systemic reaction after sting challenge [9, 19, 49]. The confrontation of the demonstrated overall efficacy for in vitro tests with results provided from the trials based on the sting challenge indicates that a further critical evaluation of recently developed diagnostic methods is required. The lower challenge test sensitivity as compared to the in vitro ones and the fact that only $75 \%$ of adults with a history of anaphylactic sting reactions develop systemic symptoms when re-stung might lead to the suggestion that actual cellular tests cannot distinguish individuals who will develop a self-limitation of clinical sensitivity $[1,7,19]$. In other words, a false positive result for in vitro tests among subjects with negative usual test responses is not excluded, because only a significantly lower proportion of them experiences a systemic reaction when re-stung or during the sting challenge [7, 19]. Therefore, cellular tests might be rather detectors of previous sensitization than predictors of its persistence. Consequently, the presence of systemic reaction after natural or provoked exposure to sting should drive the SIT initiation in these cases. Moreover, the low rate of systemic reactions after sting challenge among patients with negative standard tests results may decrease the cost-efficiency relationship for the subsequent introduction of cellular tests.

In developing countries, the allergology practitioners could be more frequently faced with negative venom ST and/or specific IgE patient dilemma. On the first hand, in vitro/cellular tests are not in disposition, or if so, they may be very expensive for the majority of people. On the other hand, the sting challenge is often considered very dangerous because of equipment deficiencies in intensive care units. In these circumstances, SIT introduction could be considered by the allergologist when the culprit insect is clearly identified by the subject who experienced systemic reaction independently to the outcome of the usual tests. This strategy may be supported by the fact that SIT may induce T cell anergy through IL-10 release in a proportion of allergic subjects, even if specific IgE to offending allergens remain undetected [53, 54]. The possible target of this therapeutic strategy might be adults with severe or repeated systemic "non IgE-mediated" reactions after a hymenoptera sting, because of the higher risk for subsequent life-threatening events [7, 54]. However, further studies are needed for the elaboration of suitable therapeutic strategies with respect to this issue. While SIT introduction in such patients should be evaluated for the cost-efficiency, much effort is required in the improving of diagnostic capacities, especially in developing countries.

\section{CONCLUSION}

If hymenoptera sting patients have unequivocal and concordant specific IgE and ST results, no additional tests are mandatory [1]. In the remaining patients, diagnosis can be complicated by entirely negative ST and specific IgE results $[1,9,19]$. Even reported more frequently as previously thought, current practice guidelines do not adequately address the management and evaluation of these subjects $[6,7$, 9]. Despite the limited number of relevant published reports related to this issue, modified working guidelines are needed based on the current evidence. Apart from an allergy exclusion, this evidence demonstrates that BAT, CAST-ELISA, Western blot, and optionally, the sting challenge constitute complementary diagnostic instruments that contribute to the potentially life-saving SIT initiation [1-3, 6, 16-19]. In contrast, ST with dialyzed HV (except fire ants) could be considered as part of the standard diagnostic regimen [15]. While a negative ST and/or in vitro assay result should not be considered as guarantee of safety, patients with a suspected higher risk should be counseled about avoidance strategies, use of epinephrine auto-injectors, and the emergent and follow-up care of the acute allergic reaction $[9,15]$. Furthermore, the management of patients with a positive history and a negative diagnostic result requires clinical judgment and ongoing research [9]. Especially in developing countries, SIT introduction may be considered (together with epinephrine auto-injectors use) at least among adults with important clinical history for HV allergy, independently to the outcome of conventional tests (see the Fig. 1).

ABBREVIATIONS LIST
BAT $=$ Basophil Activation Test
BHR $=$ Basophil Histamine Releasing test
BSACI $=$ British Society for Allergy \& Clinical Immu-
nology
CAST $=$ Cellular Antigen Stimulation Test
CD $=$ Cluster of Differentiation
ELISA $=$ Enzyme-Linked Immuno-Sorbent Assay
HV $=$ Hymenoptera Venom
IL $=$ Interleukin
Ig $=$ Immunoglobulin
LT $=$ Leukotriene
RAST $=$ Radio-Allergen Sorbent Test
RIA $=$ Radio-Immuno Assay
SIT $=$ Specific Immunotherapy
ST $=$ Skin Test(s)

\section{REFERENCES}

[1] Ebo DG, Hagendorens MM, Bridts CH, De Clerck LS, Stevens WJ. Hymenoptera venom allergy: taking the sting out of difficult cases. J Invest Allergol Clin Immunol 2007; 17(6): 357-60.

[2] Eberlein-König B, Rakoski J, Behrendt H, Ring J. Use of CD63 expression as marker of in vitro basophil activation in identifying the culprit in insect venom allergy. J Invest Allergol Clin Immunol 2004; 14(1): 10-6.

[3] Scherer K, Weber JM, Jermann TM, et al. Cellular in vitro assays in the diagnosis of hymenoptera venom allergy. Int Arch Allergy Immunol 2008; 146(2): 122-32.

[4] Hoffman DR. Fatal reactions to hymenoptera stings. Allergy Asthma Proc 2003; 24(2): 123-7.

[5] Tavares B. [Hymenoptera venom allergy - new diagnostic methods]. Acta Med Port 2005; 18(6): 445-51. [in Portuguese]

[6] Biló BM, Rueff F, Mosbech H, Bonifazi F, Oude-Elberink JN. The EAACI Interest Group on Insect Venom Hypersensitivity*. Diagnosis of Hymenoptera venom allergy. Allergy 2005; 60(11): 133949.

[7] Bonifazi F, Jutel M, Biló BM, Birnbaum J, Muller U. EAACI interest group on insect venom hypersensitivity. Prevention and treatment of hymenoptera venom allergy: guidelines for clinical practice. Allergy 2005; 60(12): 1459-70. 
[8] Zidarn M, Kosnik M, Drinovec I. Anaphylaxis after hymenoptera sting without detectable specific IgE. Acta Dermatovenerol Alp Panonica Adriat 2007; 16(1): 31-3.

[9] Golden DB, Tracy JM, Freeman TM, Hoffman DR. Insect Committee of the American Academy of Allergy, Asthma and immunology. Negative venom skin test results in patients with histories of systemic reaction to a sting. J Allergy Clin Immunol 2003; 112(3): 495-8.

[10] Diwakar L, Noorani S, Huissoon AP, Frew AJ, Krishna MT. Practice of venom immunotherapy in the United Kingdom: a national audit and review of literature. Clin Exp Allergy 2008; 38(10): 1651-8.

[11] Nittner-Marszalska M. [New variants of diagnostic procedures and therapeutic methods in insect venom allergy]. Pol Merkur Lekarski 2003; 14(84): 700-2. [in Polish]

[12] Mothes N, Valenta R, Spitzauer S. Allergy testing: the role of recombinant allergens. Clin Chem Lab Med 2006; 44(2): 125-32.

[13] Müller UR, Johansen N, Petersen AB, Fromberg-Nielsen J, Haeberli G. Hymenoptera venom allergy: analysis of double positivity to honey bee and Vespula venom by estimation of $\operatorname{IgE}$ antibodies to species-specific major allergens Api m1 and Ves v5. Allergy 2009; 64(4): 543-8.

[14] Müller UR. New developments in the diagnosis and treatment of hymenoptera venom allergy. Int Arch Allergy Immunol 2001; 124(4): 447-53.

[15] Golden DB, Kelly D, Hamilton RG, Wang NY, Kagey-Sobotka A. Dialyzed venom skin tests for identifying yellow jacket-allergic patients not detected using standard venom. Ann Allergy Asthma Immunol 2009; 102(1): 47-50.

[16] Sturm GJ, Böhm E, Trummer M, Weiglhofer I, Heinemann A, Aberer W. The CD63 basophil activation test in hymenoptera venom allergy: a prospective study. Allergy 2004; 59(10): 1110-7.

[17] Sainte-Laudy J, Sabbah A, Drouet M, Lauret MG, Loiry M. Diagnosis of venom allergy by flow cytometry. Correlation with clinical history, skin tests, specific $\operatorname{IgE}$, histamine and leukotriene $\mathrm{C} 4$ release. Clin Exp Allergy 2000; 30(8): 1166-71.

[18] Zollner TM, Spengler K, Podda M, Ergezinger K, Kaufmann R, Boehncke WH. The Western blot is a highly sensitive and efficient technique in diagnosing allergy to wasp venom. Clin Exp Allergy 2001; 31(11): 1754-61.

[19] Golden DB, Kagey-Sobotka A, Norman PS, Hamilton RG, Lichtenstein LM. Insect sting allergy with negative venom skin test responses. J Allergy Clin Immunol 2001; 107(5): 897-901.

[20] Kontou-Fili K. Patients with negative skin tests. Curr Opin Allergy Clin Immunol 2002; 2(4): 353-7.

[21] Nittner-Marszalska M, Małolepszy J, Medrala W. [Evaluation of diagnostic value skin test, venom specific antibodies against IgE, and basophil histamine release test in hymenoptera allergy]. Pneumonol Alergol Pol 1993; 61(7-8): 346-51. [in Polish]

[22] Rieger-Ziegler V, Rieger E, Kränke B, Aberer W. Hymenoptera venom allergy: time-course of specific IgE concentrations during first weeks after a sting. Int Arch Allergy Immunol 1999; 120(2): 166-8.

[23] Goldberg A. Variability of venom skin tests. Curr Opin Allergy Clin Immunol 2007; 7(4): 342-5.

[24] Hamilton RG. Diagnostic methods for insect sting allergy. Curr Opin Allergy Clin Immunol 2004; 4(4): 297-306.

[25] Kleine-Tebbe J, Erdmann S, Knol EF, MacGlashan DW Jr, Poulsen LK, Gibbs BF. Diagnostic tests based on human basophils: potentials, pitfalls and perspectives. Int Arch Allergy Immunol 2006; 141(1): 79-90.

[26] Sturm GJ, Schuster C, Kranzelbinder B, Wiednig M, Groselj-Strele A, Aberer W. Asymptomatic sensitization to hymenoptera venom is related to total immunoglobulin E levels. Int Arch Allergy Immunol 2009; 148(3): 261-4.

[27] More DR, Kohlmeier RE, Hoffman DR. Fatal anaphylaxis to indoor native fire ant stings in an infant. Am J Forensic Med Pathol 2008; 29(1): 62-3.

[28] Fernández-Meléndez S, Miranda A, García-González JJ, Barber D, Lombardero M. Anaphylaxis caused by imported red fire ant stings in Málaga, Spain. J Invest Allergol Clin Immunol 2007; 17(1): 489.

[29] Jappe U, Raulf-Heimsoth M, Hoffmann M, Burow G, HübschMüller C, Enk A. In vitro hymenoptera venom allergy diagnosis: improved by screening for cross-reactive carbohydrate determinants and reciprocal inhibition. Allergy 2006; 61(10): 1220-9.
[30] Kochuyt AM, Van Hoeyveld EM, Stevens EA. Prevalence and clinical relevance of specific immunoglobulin $\mathrm{E}$ to pollen caused by sting- induced specific immunoglobulin $\mathrm{E}$ to cross-reacting carbohydrate determinants in Hymenoptera venoms. Clin Exp Allergy 2005; 35(4): 441-7.

[31] Eberlein-König B, Ring J. Diagnosis of IgE-mediated hymenoptera venom anaphylaxis in patients with negative skin tests and negative RAST using cellular in vitro tests. J Allergy Clin Immunol 2004; 113(6): 1223-4.

[32] Schiavino D, Nucera E, Pollastrini E, et al. Specific ultrarush desensitization in Hymenoptera venom-allergic patients. Ann Allergy Asthma Immunol 2004; 92(4): 409-13.

[33] Ebo DG, Hagendorens MM, Bridts CH, De Clerck LS, Stevens WJ. Sensitization to cross-reactive carbohydrate determinants and the ubiquitous protein profilin: mimickers of allergy. Clin Exp Allergy 2004; 34(1): 137-44

[34] Golden DB. Discontinuing venom immunotherapy. Curr Opin Allergy Clin Immunol 2001; 1(4): 353-6.

[35] Hemmer W, Focke M, Kolarich D, et al. Antibody binding to venom carbohydrates is a frequent cause for double positivity to honeybee and yellow jacket venom in patients with stinging-insect allergy. J Allergy Clin Immunol 2001; 108(6): 1045-52.

[36] Stern A, Wüthrich B, Müllner G. Successful treatment of occupational allergy to bumblebee venom after failure with honeybee venom extract. Allergy 2000; 55(1): 88-91.

[37] Golden DB, Kagey-Sobotka A, Lichtenstein LM. Survey of patients after discontinuing venom immunotherapy. J Allergy Clin Immunol 2000; 105(2 Pt 1): 385-90.

[38] Reimers AR, Weber M, Müller UR. Are anaphylactic reactions to snake bites immunoglobulin E-mediated? Clin Exp Allergy 2000; 30(2): 276-82.

[39] Przybilla B, Ruëff F. [Desensitization of allergy to hymenoptera venoms]. Wien Med Wochenschr 1999; 149(14-15): 421-8. [in German]

[40] Lerch E, Müller UR. Long-term protection after stopping venom immunotherapy: results of re-stings in 200 patients. J Allergy Clin Immunol 1998; 101(5): 606-12.

[41] Egner W, Ward C, Brown DL, Ewan PW. The frequency and clinical significance of specific IgE to both wasp (Vespula) and honeybee (Apis) venoms in the same patient. Clin Exp Allergy 1998; 28(1): 26-34.

[42] Golden DB, Kwiterovich KA, Kagey-Sobotka A, Lichtenstein LM. Discontinuing venom immunotherapy: extended observations. J Allergy Clin Immunol 1998; 101(3): 298-305.

[43] Golden DB. Insect sting allergy and venom immunotherapy: a model and a mystery. J Allergy Clin Immunol 2005; 115(3): 43947.

[44] Kränke B, Sturm G, Aberer W. Negative venom skin test results and mastocytosis. J Allergy Clin Immunol 2004; 113(1): 180-1.

[45] Volcheck GW. Hymenoptera (apid and vespid) allergy: update in diagnosis and management. Curr Allergy Asthma Rep 2002; 2(1): 46-50.

[46] Novembre E, Cianferoni A, Bernardini R, et al. Anaphylaxis in children: clinical and allergologic features. Pediatrics 1998; 101(4): E8.

[47] Antonicelli L, Bilò MB, Bonifazi F. Epidemiology of hymenoptera allergy. Curr Opin Allergy Clin Immunol 2002; 2(4): 341-6.

[48] Hamilton RG. Diagnosis of hymenoptera venom sensitivity. Curr Opin Allergy Clin Immunol 2002; 2(4): 347-51.

[49] Reisman RE. Insect sting allergy: The dilemma of the negative skin test reactor. J Allergy Clin Immunol 2001; 107(5): 781-2.

[50] Cahen YD, Maly FE, Wüthrich B. [Cellular antigen stimulation test (CAST) - applicability in the diagnosis of insect toxin allergies]. Schweiz Med Wochenschr 1997; 127(1-2): 5-11. [in German]

[51] Radermecker MF, Leclercq MD, Mariz SD, Louis RE. Basophil releasability in patients with hymenoptera venom allergy. Int Arch Allergy Immunol 1993; 101(3): 283-7.

[52] Goldberg A, Confino-Cohen R, Mekori YA. Deliberate hymenoptera sting challenge as a diagnostic tool in highly selected venomallergic patients. Ann Allergy Asthma Immunol 1995; 75(1): 30-2.

[53] Akdis CA, Blaser K. IL-10-induced anergy in peripheral T cell and reactivation by microenvironmental cytokines: two key steps in specific immunotherapy. FASEB J 1999; 13(6): 603-9. 
[54] Mingomataj EÇ. Favorable hymenoptera sting reactions during childhood may have enabled transmission of responsible allergic

genotype into generations. Med Hypotheses 2006; 67(2): 327-32.

Received: November 02, 2009

Revised: January 14, 2010

Accepted: January 16,2010

(c) Mingomataj and Bakiri; Licensee Bentham Open.

This is an open access article licensed under the terms of the Creative Commons Attribution Non-Commercial License (http://creativecommons.org/licenses/by-nc/3.0/) which permits unrestricted, non-commercial use, distribution and reproduction in any medium, provided the work is properly cited. 